\title{
Research on the selection algorithm of control nodes of emergency communication system in the coal mine

\author{
Xiaoqian You, Sicheng Lu
}

Chongqing university of posts and telecommunications, Chongqing ,China

Keyword: coal mine; emergency communication; control nodes; competitive algorithm

\begin{abstract}
Emergency mobile communication system is an important means of emergency rescue. To assess network performance rapidly under different scenarios is the important basis for researching the organization and application of emergency mobile communication system, which plays an important role for making effec-tive emergency plans and making full use of emergency communication system. In this paper, a control node optimization selection algorithm is proposed based on the idea of graph theory in order to solve the problem of control nodes selection in the mine emergency communication. This algorithm will be set up to the least number of controlled nodes and the maximum time delay as the optimization goal to choose a certain number of control nodes. the experimental results showed that the algorithm is effective.
\end{abstract}

\section{INTRODUCTION}

\subsection{Research of status}

The mine production process is very complex, mine suffer natural disasters under the harsh environmental conditions and some factors. When mine accident occurs, it is urgently needed to solve problems that how to quickly and efficiently to inform underground staff to evacuate as soon as possible and inform the ground operations command center in the first place.

When major natural disasters or a sudden public events, fixed wired and wireless communication network of cellular base station is easy to damage, make the emergency site user groups can not be effectively access networks or communicate with the rear of the command center to establish a reliable connection, and regular communication network to laying and want to spend a lot of manpower and material resources and time, which can't adapt to the requirement of emergency communication rapid disposal.

After losing connected to the core network gateway, the interconnection between the base station and the base station will create a new ad-hoc network, emergency communication system is collect the related information timely and efficiently in case of emergency.

\subsection{The solution and existing problems}

The solution of problem of digital mine emergency communication including the following:

(1) The Zigbee technology[5][9] is applied to underground emergency communication, using the SDR wireless technology through automatic cloth device to quickly establish a wireless communication channels, and extend to the rescue ground zero by wireless relay way.But Zigbee technology has an obvious flaw: with the transmission distance increasing, the single node can work stability only tens of meters, making requires a number of nodes to ensure communication link can establish successful. all of this lead to great inconvenience work to do.

(2) WIFI base station[5] using knapsack. Since the power control effect WIFI is limited, so we use the piggyback WIFI to solute the higher power requirements, which rescue sustainability challenges.

(3)Using Wireless Mesh Network scheme(WMN)[1],which is from Ad Hoc network, and is based on the Ad Hoc network derived from large capacity, high speed and easy deployment of WMN.Wireless Mesh has some shortcomings: First, the problems on interoperability. Wireless Mesh Network is still not a unified standards in network technology, to solve this problems, the ul- 
timately standards of technology is needed. Second, since the data Mesh network through intermediate nodes in multi-hop, each hop will bring some delay. With the expansion of wireless Mess network size, the more hops, the greater total accumulated delay. Some application, such as voice or streaming applications, could face long delays and unacceptable problem.

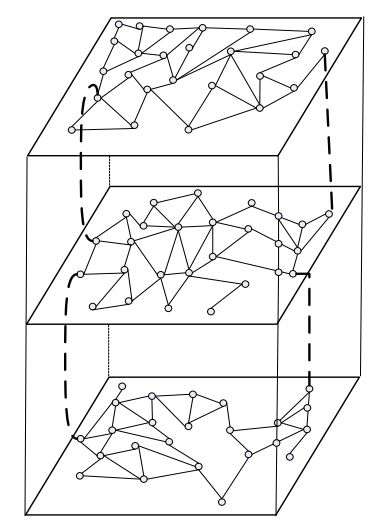

Figure 1 the distribution structure of mine nodes

\section{ALGORITHM DESCRIPTION}

\subsection{The problem of control node selection}

When emergencies occur in Figure 2(b) as below. A new ad hoc network, select a off-network gateway to collect control information once losing the core network gateway connection. The facing problem is: 1) to determine the number of nodes; 2)to determine the position of nodes.

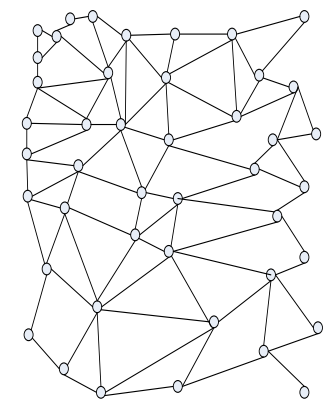

(a) before

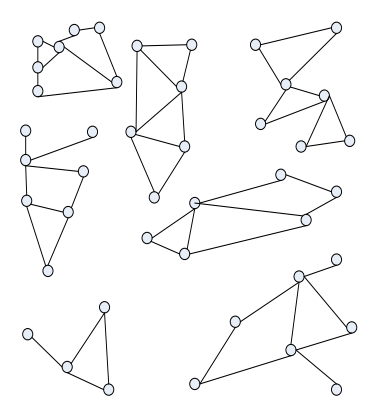

(b) after

Figure 2.The distribution of communication nodes in mine before and after the accident

\subsection{The algorithm analysis of control node}

Currently, most research focuses on head selection algorithm network in two-dimensional. The description of the algorithm in article [4] :

1) every mobile device saves the current cluster heads of information;

2) when using mobile devices to move to a new node, it notifies the current cluster head;

3 ) the current cluster heads will determine whether delay and registration costs be satisfied;

4) if the cluster will accept and satisfy the update data, otherwise the new node registered directly to gateway, become mobile cluster heads.

Defect: if the number of new cluster heads too many, how to detect the cluster head is a problem.

Article [3] algorithm is based on $4 \mathrm{~d}$ model, in the know a number of alternatives under the condition of the location of the control node, select the control node method.Because of its location control node is known, if applied to the mine emergency communication system lacks the rationality of the actual situation. 


\subsection{The solution of actual situation}

When the network is damaged, underground stations will find that with the total server disconnected, happens to take off the network state;Then the emergency communication system start, need to base station as a network gateway.

So the main problem is that to select the control node in a single connected network which in each control node can cover as many nodes (base station), as well as the control node load equilibrium network.

Set two conditions:

(1) to select a minimum of the number of nodes, setting the control node and the control of maximum transmission delay between nodes $D_{\text {max }}$;

(2) in order to prevent the load, set the maximum number of connections to set the node $X$.

Control node selection algorithm competition(CNSCA):

1) calculate the number of the time delay between very node and its connection nodes no more than $D_{\max }$ in the network topology;

2) choose the most number of the node as the first control $P_{1}$;

3 ) if the number of the control nodes $P_{1}$ connected is greater than $\mathrm{X}$, choose just $\mathrm{X}$ (according to the length of the delay ), other abandoned. Otherwise go to 4)

4) eliminate $P_{1}$ and its collection $R_{1}$ from the topology, continue to look for the second node;

5) repeat the second, three steps, to select the most number, as the second node $P_{2}$ and its collection $R_{2}$;

6) continue to weed out the second node and the set, repeat the second, three steps, looking for the rest of the control node, until the network without the rest of the nodes.

\subsection{Algorithm description}

1. Generate $G=(V, E)$;

2. While $(V !=\phi)$;

3. $\quad\{$ For $(\mathrm{i}=0 ; \mathrm{i}<\mathrm{V}$.Size $; \mathrm{i}++)$

4. $\quad$ For $(\mathrm{j}=0 ; \mathrm{j}<\mathrm{V}$.Size; $\mathrm{j++})$

5. $\quad\left\{\right.$ If( $\left(\mathrm{h}_{i j} \leq \mathrm{D}_{\text {max }},\right)$

6. $\left\{N \geq 0, N_{i}++\right.$;

7. $\left.\left.R_{i}=R_{i} \cup\left\{r_{j}\right\} ;\right\}\right\}$

8. If $N_{i}$ is $\max (N), N_{i} \leq X$

9. $P_{i}=P_{i} \cup\left\{r_{i}\right\}$;

10. Delete $R_{i}$ from G;

11. $\}$

12. Repeat 2-11;

13. $P=\left\{P_{1}, P_{2}, \ldots, P_{i}\right\}$

14. Output $P$

Algorithm output the set of $\mathrm{P}$ is the set of all the control node. Algorithm of line 3-7 calculated the delay no more $\mathrm{D}_{\max }$ than between each node and the adjacent nodes. Line 8, 9 means to select the most number of the nodes, and the number is no more than X. Line 10-11 said they would choose a collection of nodes and their connection is removed from the topology. Line 12 said repeat the above step. Line 13, 14said output the set of all the control node P. 


\section{EXPERIMENTAL ANALYSIS}

To further verify and compare the effectiveness of CNSCA algorithm, this paper adopted the same experimental parameters as the literature [3], using MATLAB to carry out the simulation experiment. Table 1 shows the three parameters of network topology, $T_{\max }$ is the maximum time delay between two nodes, $T_{\text {avg }}$ is the average delay between two nodes.

Table 1 parameters of the experiment network topology

\begin{tabular}{|c|c|c|c|}
\hline $\begin{array}{l}\text { serial num- } \\
\text { ber }\end{array}$ & $\begin{array}{l}\text { Number } \\
\text { of nodes }\end{array}$ & $T_{\max }$ & $T_{a v g}$ \\
\hline 1 & 138 & 137 & 17.43 \\
\hline 2 & 104 & 54 & 7.82 \\
\hline 3 & 87 & 47 & 6.25 \\
\hline
\end{tabular}

Set $D_{\max }$ the value of the 5,10,15,25,30 respectively. As shown in figure 3 when the value of the maximum time delay increases, the number of control nodes corresponding to reduce, so it shows the CNSCA algorithm is effective.

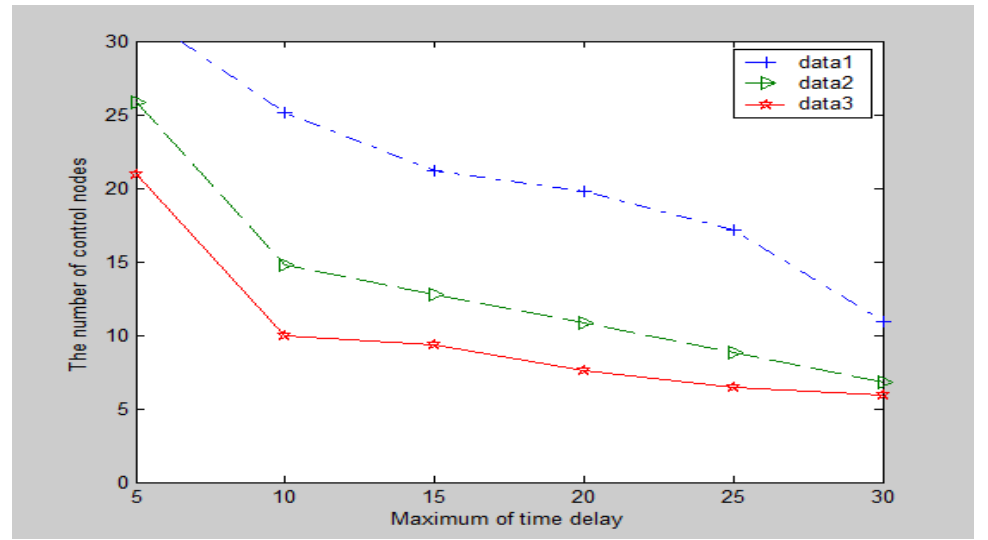

Figure 3. Relationship between control nodes and maximum delay

The CNSCA algorithm comparing with DCNSA algorithm in ref. [3] The two algorithms are compared in the maximum time delay under the same control the number of nodes on the basis of three network topology in table 1. 


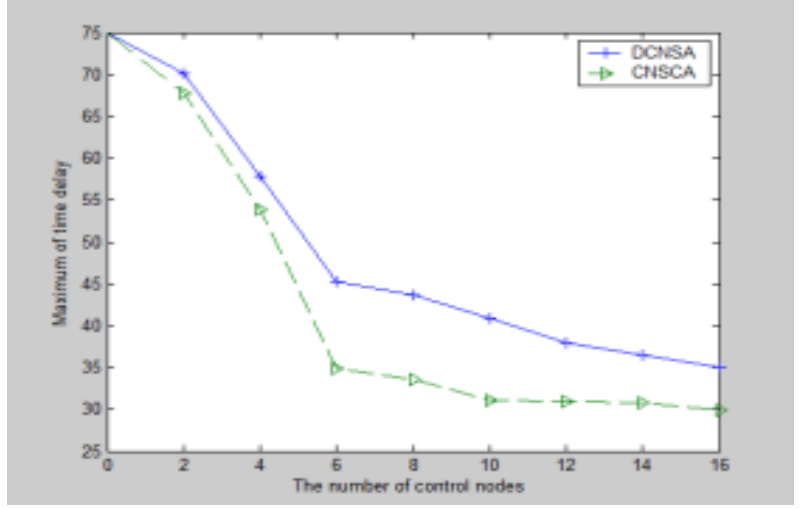

(a)

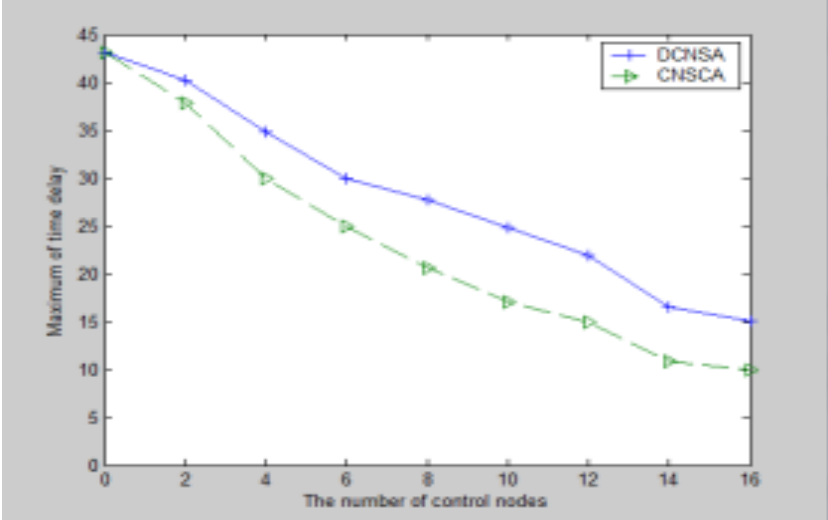

(b)

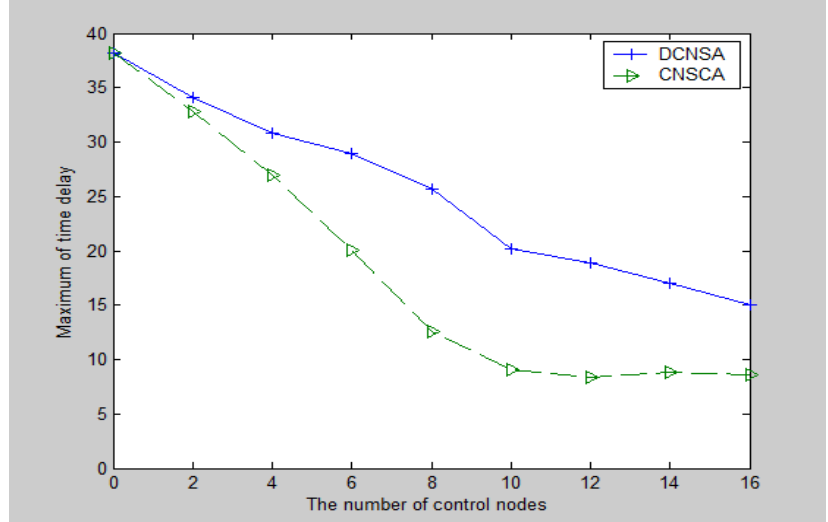

(c)

Figure 4.CNSCA algorithm compared with DCNSA algorithm of maximum delay.(a)data 1,(b)data 2,(c)data 3.

As shown in figure 4, CNSCA algorithm of maximum delay is smaller than DCNSA algorithm under the condition of the same control nodes compared the results of three groups of data. 


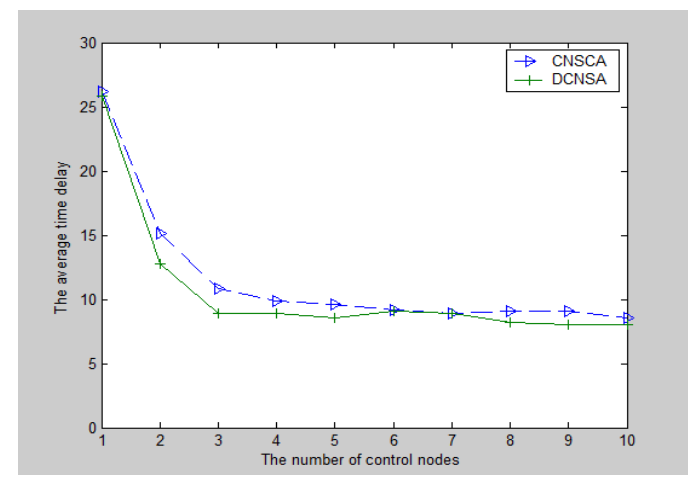

(e)

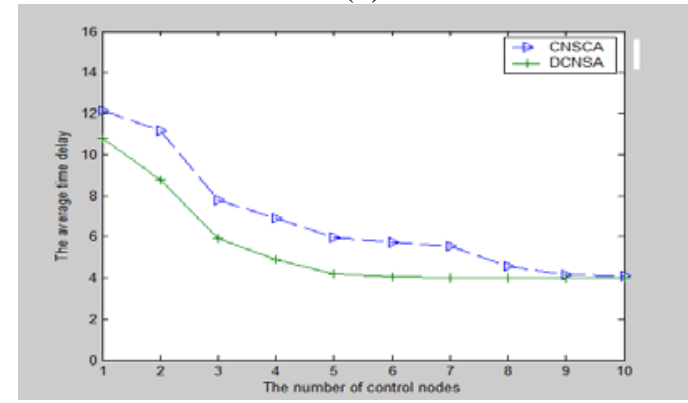

(f)

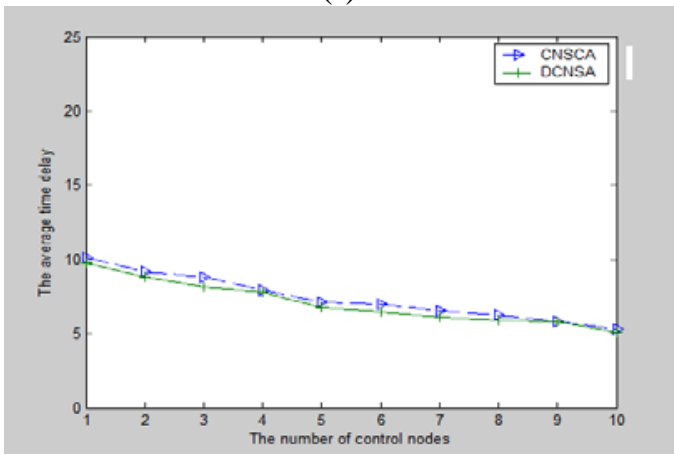

(g)

Figure 5.CNSCA algorithm compared with DCNSA algorithm of the average time delay.(e)data 1,(f)data 2,(g)data 3.

As shown in figure 5, compare the results of three groups of data, you can see that in the case of the same number of control nodes, the average delay of CNSCA algorithm slightly larger, but the two algorithms of average delay are very close.

In order to obtain all information more efficiently, we should give priority to reduce the number of control nodes, so the experimental results show that the CNSCA algorithm performance is better than DCNSA algorithm when maximum of time delay between the control nodes and control the nodes no more than $D_{\max }$ because of the special environment in mine.

\section{CONCLUSION}

This paper proposes a control node CNSCA competition optimal selection algorithm based on the idea of graph theory in view of the mine emergency communication under the selection of control node. This algorithm will be set up to control the least number of nodes and the maximum time delay as the optimization goal, both to ensure that the load capacity of the base station and the realtime performance of control; the experimental results show that CNSCA algorithm is effective. The next step is to optimize the algorithm,solve and optimize the problem of how to select new control out of control node when control nodes go wrong. 


\section{REFERENCES}

[1] Bassam Aoun, Raouf Boutaba, Senior Member.Gateway Placement Optimization in Wireless Mesh Networks With QoS Constraints[J]. IEEE JOURNAL ON SELECTED AREAS IN COMMUNICATIONS,VOL.24,NO.11,NOVEMBER 2006.

[2] DING Enjie , HUANG Yanqiu, HUANG He, YU Wanli, LIU Xuerui, A Linear-hierarchy WSN Topology Used in Coal Face[C].4th International Conference on New Trends in Information Science and Service Science (NISS), 2010

[3] Iqbal H, Znati T. Distributed control plane for 4D architecture[C].Proceedings of IEEE Global Communications Conference. Washington DC, USA, 2007: 1901-1905.

[4] Nizar Bouabdallah, Rami Langar, and Raouf Boutaba,Design and Analysis of Mobility-Aware Clustering Algorithms for Wireless Mesh Networks[J].IEEE/ACM TRANSACTIONS ON NETWORKING,VOL.18,NO.6,DECEMBER 2010.

[5] Pan Tao, Liu Xiaoyang, Hybrid Wireless Communication System Using ZigBee and WiFi Technology in the Coalmine Tunnels [C].Measuring Technology and Mechatronics Automation (ICMTMA),2011 Third International Conference on, Vol 2,pp 340-343,2011.

[6] Rezgui J, Hafid A, Gendreau M. Distributed admission control in wireless mesh networks: models,algorithms,and,evaluation[C].IEEETransactions on Vehicle Technology 2010;59(3):1459-73.

[7] R. Liu, I. J. Wassell, and K. Soga. Relay Node Placement for Wireless Sensor Networks Deployed in Tunnels[C]. In Proc. IEEE Int'l. Conf. WiMob, pages 144-150, 2010.

[8] Shan-Shan Ma, Jian-Sheng Qian .A Topology Control Algorithm for mobile Nodes Used in Coal Face[C],7th International Conference on Wireless Communications,Networking and Mobile Computing (WiCOM), 2011.

[9] Zhigao Liu and Chunwen Li.A coal mine personnel global positioning system based on wireless sensor networks [C],Proceedings of the 8thWorld Congress on Intelligent Control and Automation July 6-9 2010. 